\title{
The 9th Symposium on Future Directions in Information Access
}

\author{
Ingo Frommholz ${ }^{10000-0002-5622-5132]}$, Haiming Liu ${ }^{10000-0002-0390-3657]}$, and \\ Yashar Moshfeghi ${ }^{2}[0000-0003-4186-1088]$ \\ 1 University of Bedfordshire, Luton, UK \\ \{ingo.frommholz, haiming.liu\}@beds.ac.uk \\ 2 University of Strathclyde, Glasgow, Scotland \\ yashar.moshfeghi@strath.ac.uk
}

\section{The Symposium and its objectives}

The 9th PhD Symposium on Future Directions in Information Access (FDIA) was held in conjunction with the 12th European Summer School on Information Retrieval in Milan, Italy. The symposium aims to provide a forum for early-stage researchers such as PhD students, to share their research and interact with each other and senior researchers in an informal and relaxed atmosphere. The symposium provides an excellent opportunity for the participants to promote their work and obtain experience in presenting and communicating their research. The participants will learn about different topics in the area of information access and retrieval, receive feedback on their work, meet lots of peers and hear inspiring talks.

The symposium is about future directions because we want to encourage submissions that focus on early research such as pilot studies, presenting challenges and future opportunities, conceptual and theoretical work, and the contributions from doctoral work. The theme of the symposium is information access because it captures the broader ideas of information retrieval, storage and management to include interaction and usage.

The FDIA Symposium series is held in cooperation with the British Chartered Institute for IT's Information Retrieval Specialist Group (BCS-IRSG). The FDIA symposium is mainly tuned to $\mathrm{PhD}$ students, researchers new to the field, and postdoctoral researchers. The objectives are:

- To provide an accessible forum for early-stage researchers to discuss their research and/or projects.

- To help foster formative and tentative research ideas.

- To encourage discussion and feedback.

- To share outcomes of doctoral work.

- To network with peers and senior researchers.

Copyright (c) 2019 for this paper by its authors. Use permitted under Creative Commons License Attribution 4.0 International (CC BY 4.0). FDIA 2019, 17-18 July 2019, Milan, Italy. 
We invited submissions on formative research ideas which present a summary of early-stage researchers' work, such as initial empirical findings/pilot studies; explore conceptual and/or theoretical models; and/or describe current challenges and opportunities. In particular, submissions focusing on new directions and emerging work in information access and retrieval that potentially create discussion and provoke reaction were strongly encouraged.

\section{Previous Symposiums}

FDIA 2019 is the 9th in the series of FDIA symposiums. The previous symposiums were held in Tianjin, China in 2018 (with ICTIR 2018), Barcelona, Spain in 2017 (with ESSIR 2017); Thessaloniki, Greece in 2015 (with ESSIR 2015); Granada, Spain in 2013 (with ESSIR 2013); Koblenz, Germany in 2011 (with ESSIR 2011), Padova, Italy in 2009 (with ESSIR 2009); London, UK in 2008, and Glasgow, UK in 2007 (with ESSIR 2007). They have provided an entertaining and exciting forum for early-stage researchers for sharing new research ideas.

\section{Areas of research}

Areas of research include, but are not limited to:

- Information Retrieval Theory

- Human-Computer Interaction and Information Retrieval, Interactive IR

- Collaborative Information Seeking and Searching

- Learning to Rank

- Neural IR

- Multimedia and Multimodal IR

- Recommender Systems

- Databases + IR

- Semantic Search, Social Search

- Enterprise Search

- Web IR

- Clustering and Categorisation

- Conversational Agents and Knowledge Graphs

- IR Applications (e.g. Digital Humanities, e-Learning, News IR, IR and Bibliometrics, etc.)

\section{Submissions}

The submissions were peer-reviewed by our Programme Committee members. From the 25 submissions we received, seven were selected for oral presentation and 12 as a poster presentation. These proceedings contain the full papers of each submission presented at the symposium, either as a talk or a poster. 


\section{General Chairs and Programme Committee}

We would like to thank our general chairs and ESSIR 2019 organisers, Gabriella Pasi (University of Milan Bicocca), Maristella Agosti (University of Padua) and Nicola Ferro (University of Padua) for their neverending support to make FDIA 2019 happen. We further like to thank our programme committee and student mentors for their valuable help:

- Ricardo Baeza-Yates, NTENT \& Northeastern University at SV, USA

- Guillaume Cabanac, University of Toulouse

- Berkant Barla Cambazoglu, RMIT University

- W. Bruce Croft, University of Massachusetts

- Tess Crosbie, University of Bedfordshire

- Juan M. Fernández-Luna, University of Granada

- Lorraine Goeuriot, Laboratoire d'informatique de Grenoble

- Julio Gonzalo, UNED

- Jiafeng Guo, Chinese Academy of Sciences

- Preben Hansen, Stockholm University

- Claudia Hauff, TU Delft

- Diane Kelly, University of Tennessee

- Claus-Peter Klas, GESIS - Leibniz Institute for Social Sciences

- Udo Kruschwitz, University of Regensburg

- Andrew Macfarlane, City, University of London

- Philipp Mayr, GESIS - Leibniz Institute for Social Sciences

- Henning Müller, HES-SO Valais

- Tony Russell-Rose, UXLabs

- Ingo Schmitt, Technical University Cottbus

- Dawei Song, The Open University

- Nicola Tonellotto, ISTI, CNR

- Vishwa Vinay, Adobe Research Bangalore

- Grace Hui Yang, Georgetown University

- Guido Zuccon, Queensland University of Technology 